\title{
Multiplicative Calculus in Biomedical Image Analysis
}

\author{
Luc Florack • Hans van Assen
}

Published online: 18 March 2011

(C) The Author(s) 2011. This article is published with open access at Springerlink.com

\begin{abstract}
We advocate the use of an alternative calculus in biomedical image analysis, known as multiplicative (a.k.a. non-Newtonian) calculus. It provides a natural framework in problems in which positive images or positive definite matrix fields and positivity preserving operators are of interest. Indeed, its merit lies in the fact that preservation of positivity under basic but important operations, such as differentiation, is manifest. In the case of positive scalar functions, or in general any set of positive definite functions with a commutative codomain, it is a convenient, albeit arguably redundant framework. However, in the increasingly important non-commutative case, such as encountered in diffusion tensor imaging and strain tensor analysis, multiplicative calculus complements standard calculus in a truly nontrivial way. The purpose of this article is to provide a condensed review of multiplicative calculus and to illustrate its potential use in biomedical image analysis.
\end{abstract}

Keywords Multiplicative calculus · Non-Newtonian calculus · Diffusion tensor imaging · Cardiac strain tensor analysis $\cdot$ Positivity

\section{Introduction}

Empirically acquired images are (typically) constrained to have positive values. Although often taken into considera-

\section{Florack ( $\varangle)$}

Department of Mathematics \& Computer Science and Department of Biomedical Engineering, Eindhoven University of Technology, Eindhoven, The Netherlands e-mail: L.M.J.Florack@tue.nl

H. van Assen

Department of Biomedical Engineering, Eindhoven University of Technology, Eindhoven, The Netherlands

e-mail: H.C.v.Assen@tue.nl tion in image reconstruction, positivity is rarely adopted as an a priori axiom in image analysis. Indeed, little emphasis is put on operators that preserve positivity, often for good reasons. A counterexample is a derivative operator, which does not respect positivity of its operands. If we would consider operators admissible only if they respect positivity, then the powerful machinery of standard differential calculus would no longer be at our disposal. This example suggests that insisting on positivity may indeed be too restrictive in some cases, and that one is naturally led to admit non-positive images, such as image derivatives, at least for image analysis purposes.

However, in this paper we wish to recall an alternative for standard (a.k.a. classical, additive, or Newtonian) calculus known as multiplicative calculus, first introduced by Volterra in 1887 [35]. This appears to be a natural framework for local structural analysis whenever positive functions are of interest, and admits a positivity preserving (multiplicative) differential calculus. The use of multiplicative calculus has been advocated in other contexts, such as in the theory of survival analysis and Markov processes, cf. Gill and Johansen [20]. To the best of our knowledge it has not yet received any attention in the image literature. Its potential relevance for image analysis should also encourage the mathematical community to revive this topic, and to further explore its foundations especially in the context of noncommutative matrix algebras, for which no comprehensive account seems to exist as yet.

We start by considering scalar functions $[4,21,22$, $31,34]$ and subsequently turn to matrix valued functions $[19,24,33]$. The latter are considerably more complicated as a result of the non-commutative nature of the matrix product, but it is in this context that multiplicative calculus becomes particularly interesting. (The commutative case admits trivial workarounds via standard calculus.) In image 
analysis positive matrix valued functions are for instance encountered in the context of diffusion tensor imaging and strain tensor analysis.

After a condensed summary of multiplicative calculus collected from the literature, we will demonstrate its use by a multiplicative reformulation of two existing biomedical image analysis applications, viz. multi-scale representation (or spatial regularization) of diffusion tensor images in the framework of the log-Euclidean paradigm [1, 9, 11, 28], and tensorial strain analysis in cardiac magnetic resonance imaging [12]. These examples merely serve to illustrate the potential power of multiplicative calculus. In general, multiplicative calculus should come to mind as a potentially promising tool for addressing image analysis problems whenever some sort of multiplicative process lies beneath the surface. We shall point out what these processes are in our concrete examples.

\section{Theory}

\subsection{Background Structure}

Loosely speaking, the key to understand multiplicative calculus is a formal substitution, whereby one replaces addition and subtraction by multiplication and division, respectively. As a corollary one is then led to replace multiplication in standard calculus by exponentiation in the multiplicative case, and (thus) division by exponentiation with the reciprocal exponent. However, this naive substitution principle must be made more precise, as it leads to ambiguities. For instance, due to symmetry there is no distinction between the formal roles of the factors in a product like $a x$, given $a, x \in \mathbb{R}$, leaving us in a quandary about the intentional outcome of substitution: $a x \longrightarrow x^{a}$ or $a x \longrightarrow a^{x}$ ? To properly appreciate the substitution rule one must bring in additional structure that distinguishes $a$ from $x$. To this end we consider a (suitably restricted subspace of a) vector space, $V$, with the usual structure for vector addition and scalar multiplication, but enriched with multiplication and scalar exponentiation operations. Besides dissolving ambiguities in the scalar case, this construct allows us to generalize the mechanism to non-scalar cases (functions, matrices, etc.).

The multiplicative structure adheres to the following rules: For any $u, v, w \in V$ and $\lambda, \mu \in \mathbb{R}$ :

i. $(u v) w=u(v w)$,

ii. there exists an element $1_{V} \in V$ such that $1_{V} u=u=$ $u 1_{V}$,

iii. there exists an element $u^{-1} \in V$ such that $u u^{-1}=1_{V}=$ $u^{-1} u$,

iv. $u^{\lambda \mu}=\left(u^{\lambda}\right)^{\mu}$,

v. $u^{1}=u$.
(The unit element $1_{V} \in V$ is to be distinguished from the unit scalar $1 \in \mathbb{R}$, but the disambiguating subscript will often be suppressed if no confusion is likely to arise.) The ambiguity in the substitution rule of thumb above is resolved if we prototype $a \in V$ (with in this case $V=\mathbb{R}^{+}$as a set) and $x \in \mathbb{R}$, say, so that $a^{x}$ is well-defined, but $x^{a}$ is undefined. Note that, unlike in a standard, additive vector space structure, we refrain from introducing commutativity of vector multiplication as a basic axiom. The properties $u v=v u$ and $(u v)^{\lambda}=u^{\lambda} v^{\lambda}$ may be added as additional properties expressing commutativity, if appropriate.

In the following we will initially assume commutative multiplication, until explicitly stated otherwise. We will identify $V$ with the space of appropriately chosen, positive functions, furnished with additional multiplicative structure in the usual way by defining $(f g)(x)=f(x) g(x)$, $\left(f^{\lambda}\right)(x)=(f(x))^{\lambda}$ for $f, g \in V, \lambda \in \mathbb{R}, x \in \mathbb{R}^{n}$, et cetera. (Caveat: for a function $f, f^{-1}$ indicates multiplicative inverse, i.e. $1 / f$ in the commutative case, not compositional inverse $f^{\text {inv }}$.)

\subsection{Multiplicative Differentiation}

Below it is tacitly understood that our functions (images) of interest are positive definite and smooth. In general we may define a positive definite function $f$ as a function with a codomain in which the notion of positivity is well-defined, such that $f(x)>0$ for all $x$ in its domain of definition. In this paper the codomain may be an appropriate space of positive definite square matrices, i.e. matrices with positive real eigenvalues. For the moment we will however assume that our functions of interest are scalar valued, so that no ambiguity arises with respect to the ordering of product factors (and thus the meaning of division signs in standard calculus). Furthermore, we consider the 1-dimensional case for simplicity $(n=1)$. Quotes $\left(^{\prime}\right)$ and asterisks $\left(^{*}\right)$ will be used to denote differentiation in the one-dimensional case following the standard and multiplicative definitions, respectively.

Applying the substitution principle to the definition of a standard derivative,

$f^{\prime}(x)=\lim _{h \rightarrow 0} \frac{f(x+h)-f(x)}{h}$,

produces the definition of a multiplicative derivative,

$f^{*}(x)=\lim _{h \rightarrow 0}\left(\frac{f(x+h)}{f(x)}\right)^{1 / h}$.

It is not difficult to show that $f^{*}: \mathbb{R} \rightarrow \mathbb{R}^{+}$is positive definite if $f: \mathbb{R} \rightarrow \mathbb{R}^{+}$is positive definite, and that

$\ln f^{*}(x)=(\ln f)^{\prime}(x)$, 
Fig. 1 Commuting diagram for multiplicative and standard differentiation: $f^{*}(x)=\exp \left((\ln f)^{\prime}(x)\right)$

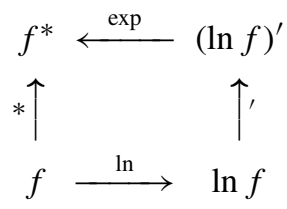

whence, more generally, using self-explanatory notation for $k$-fold differentiation,

$\ln f^{*(k)}(x)=(\ln f)^{(k)}(x)$,

cf. Fig. 1. Extension to the multivariate case is straightforward. Equation (2) combined with (4) tells us that if a function is differentiable to some order in standard sense, it is also so in multiplicative sense, vice versa.

It is clear that multiplicative calculus by itself does not provide additional instruments for analyzing (positive) images, as everything can be recast into standard form with the help of the commuting diagram, Fig. 1. Nevertheless, it may significantly simplify the analysis in some cases, which is an advantage by itself. More importantly, however-and this is our main motivation here-its generalization to the noncommutative case does provide a genuine extension that has no (obvious) standard counterpart.

\subsection{Multiplicative Integration}

Antiderivatives or indefinite integrals are introduced in multiplicative calculus as follows:

$* \int f(x)^{d t}=c F(x)$

for some constant $c \in \mathbb{R}^{+} \quad$ iff $F^{*}=f$,

in analogy with its standard counterpart:

$\int f(x) d t=F(x)+c$

for some constant $c \in \mathbb{R} \quad$ iff $F^{\prime}=f$.

Note that in the former case we denote the measure $d t$ as a formal ("infinitesimal") exponent, instead of a formal multiplier, consistent with our substitution rules.

Definite integrals can be introduced via a spatial partitioning and limiting procedure akin to the familiar Riemann sum approximation:

$* \int_{a}^{b} f(x)^{d x}=\lim _{\triangle x_{i} \rightarrow 0} \prod_{i=1}^{N} f\left(\xi_{i}\right)^{\Delta x_{i}}$

with $\xi_{i} \in\left[x_{i-1}, x_{i}\right]$ and $x_{0}=a, x_{N}=b$,

cf.

$\int_{a}^{b} f(x) d x=\lim _{\triangle x_{i} \rightarrow 0} \sum_{i=1}^{N} f\left(\xi_{i}\right) \Delta x_{i}$

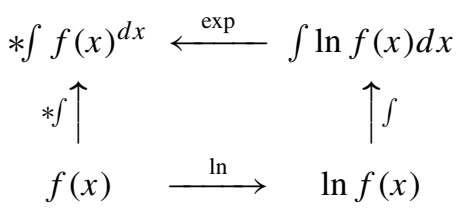

Fig. 2 Commuting diagram for multiplicative and standard antiderivation: $* \int f(x)^{d x}=\exp \left(\int \ln f(x) d x\right)$

with $\xi_{i} \in\left[x_{i-1}, x_{i}\right]$ and $x_{0}=a, x_{N}=b$,

in which $\triangle x_{i}=x_{i}-x_{i-1}$. The relationship between (5) and (7) is formalized by the following fundamental theorem of multiplicative calculus:

$* \int_{a}^{b} F^{*}(x)^{d x}=\frac{F(b)}{F(a)}$,

recall the well-known standard counterpart relating (6) and (8):

$\int_{a}^{b} F^{\prime}(x) d x=F(b)-F(a)$.

Again, by virtue of commutativity of multiplication there exists a simple one-to-one mapping between standard and multiplicative antiderivatives or integrals, cf. Fig. 2. In the non-commutative case this is no longer self-evident, as we will see in Sect. 2.8.

\subsection{Linear Functions and Linear Mappings}

Linear functions are of special interest for various purposes. In both standard and (commutative) multiplicative calculus they can be defined as those functions that have a constant derivative (i.e. we adhere to the common abuse of terminology by allowing a constant offset). This immediately yields

$f(x)=a x+b$,

$f(x)=b a^{x}$,

in standard, respectively multiplicative calculus (i.e. $f^{\prime}(x)=$ $a$, respectively $\left.f^{*}(x)=a\right)$. Thus the exponential function is the multiplicative analogue or "multiplicative linear function" of the standard linear function. Recall that in the latter case it is assumed that $f>0$, implying $a, b \in \mathbb{R}^{+}$.

In general, linear mappings are defined without offsets ( $b$ parameter in (11-12)). Again, a linear mapping $A: V \rightarrow$ $W$ in the context of multiplicative calculus obeys the same rules as in standard linear algebra, subject to aforementioned formal operator substitutions: For $u, v \in V, \lambda, \mu \in \mathbb{R}$,

$A(\lambda u+\mu v)=\lambda A(u)+\mu A(v)$,

$A\left(u^{\lambda} v^{\mu}\right)=A(u)^{\lambda} A(v)^{\mu}$, 
in the standard, respectively multiplicative case. Derivation and antiderivation provide important examples in our case. In analogy with the well-known standard results,

$(\lambda f+\mu g)^{\prime}=\lambda f^{\prime}+\mu g^{\prime}$,

$\int(\lambda f+\mu g) d x=\lambda \int f d x+\mu \int g d x$,

we have for the multiplicative case

$\left(f^{\lambda} g^{\mu}\right)^{*}=\left(f^{*}\right)^{\lambda}\left(g^{*}\right)^{\mu}$,

$* \int\left(f^{\lambda} g^{\mu}\right)^{d x}=\left(* \int f^{d x}\right)^{\lambda}\left(* \int g^{d x}\right)^{\mu}$.

\subsection{Taylor Expansions}

Analogous to the standard Taylor expansion of an analytic function,

$$
\begin{aligned}
f(x)= & \sum_{k=0}^{M} \frac{1}{k !} f^{(k)}(a)(x-a)^{k} \\
& +\frac{1}{(M+1) !} f^{(M+1)}(\xi)(x-a)^{M+1},
\end{aligned}
$$

for some $\xi$ in-between $x$ and $a$, we have in the multiplicative case

$$
\begin{aligned}
f(x)= & \prod_{k=0}^{M}\left(f^{(* k)}(a)\right)^{\frac{1}{k !}(x-a)^{k}} \\
& \times\left(f^{(*(M+1))}(\xi)\right)^{\frac{1}{(M+1) !}(x-a)^{M+1}} .
\end{aligned}
$$

In particular this leads to the linear approximation of a positive analytic function,

$$
f(x) \approx f(a) f^{*}(a)^{x-a}
$$

cf. the standard approximation

$$
f(x) \approx f(a)+f^{\prime}(a)(x-a)
$$

Multiplicative approximations (to any order) have the advantage of preserving manifest positivity, unlike the standard ones. In both cases the local approximations hold up to a small additive term (in the standard case), respectively a multiplicative factor close to unity (in the multiplicative case).

As an illustration, let us consider two intrinsically positive functions often encountered in image analysis. The sigmoidal function is given by

$f(x)=\frac{1}{1+e^{-x}}$.
Its standard and multiplicative first order Taylor approximations are given by

$f(x) \approx f_{s}(x)=\frac{1}{2}+\frac{1}{4} x$,

$f(x) \approx f_{m}(x)=\frac{1}{2} \exp \left(\frac{1}{2} x\right)$.

The former is seen to violate positivity as soon as $x \leq-2$.

As a second example, consider the standard Gaussian function,

$f(x)=\frac{1}{\sqrt{2 \pi}} \exp \left(-\frac{1}{2} x^{2}\right)$.

Due to symmetry its first order derivative is trivial at the origin, both in standard as well as multiplicative differential sense (i.e. its value is 0 , respectively 1 ). The respective second order Taylor approximations are now given by

$f(x) \approx f_{s}(x)=\frac{1}{\sqrt{2 \pi}}-\frac{1}{2 \sqrt{2 \pi}} x^{2}$,

$f(x) \approx f_{m}(x)=\frac{1}{\sqrt{2 \pi}} \exp \left(-\frac{1}{2} x^{2}\right)$.

The multiplicative approximation in fact turns out to be exact! (Cf. Sect. 2.7 to appreciate why this happens to be so.) In general one may observe that, in addition to positivity preservation, multiplicative expansions typically provide better approximations for compactly supported positive smooth filters. Such filters are abundant in image processing.

Standard and multiplicative Taylor expansions for the sigmoidal and Gaussian functions are illustrated in Figs. 3 and 4.

\subsection{Critical Points}

The following claims can be easily verified. If $f^{*}(x)>1$ then $f$ is strictly increasing at $x \in \mathbb{R}$. If $f^{*}(x)<1$ then $f$ is strictly decreasing at $x \in \mathbb{R}$. If $f^{*}(x)=1$ then $f$ has a critical point at $x \in \mathbb{R}$, viz. a local minimum if $f^{* *}(x)>1$, a local maximum if $f^{* *}(x)<1$, and an indifferent or degenerate critical point if $f^{* *}(x)=1$. This mimics the standard results, obtained by replacing multiplicative derivation by ordinary derivation, and the unit element 1 by the null element 0 in the above. These observations may provide the foundations for a multiplicative variational calculus for multiplicative energy functionals for image optimization problems, and can be easily generalized to the multivariate setting. We will not elaborate on this.

\subsection{Differential Equations}

It is well-known that many natural phenomena can be modeled in terms of ordinary or partial differential equations 
Fig. 3 Sigmoidal function and its first order Taylor expansions in standard and multiplicative sense. Positivity is manifest only in the latter case. Recall (23-25)
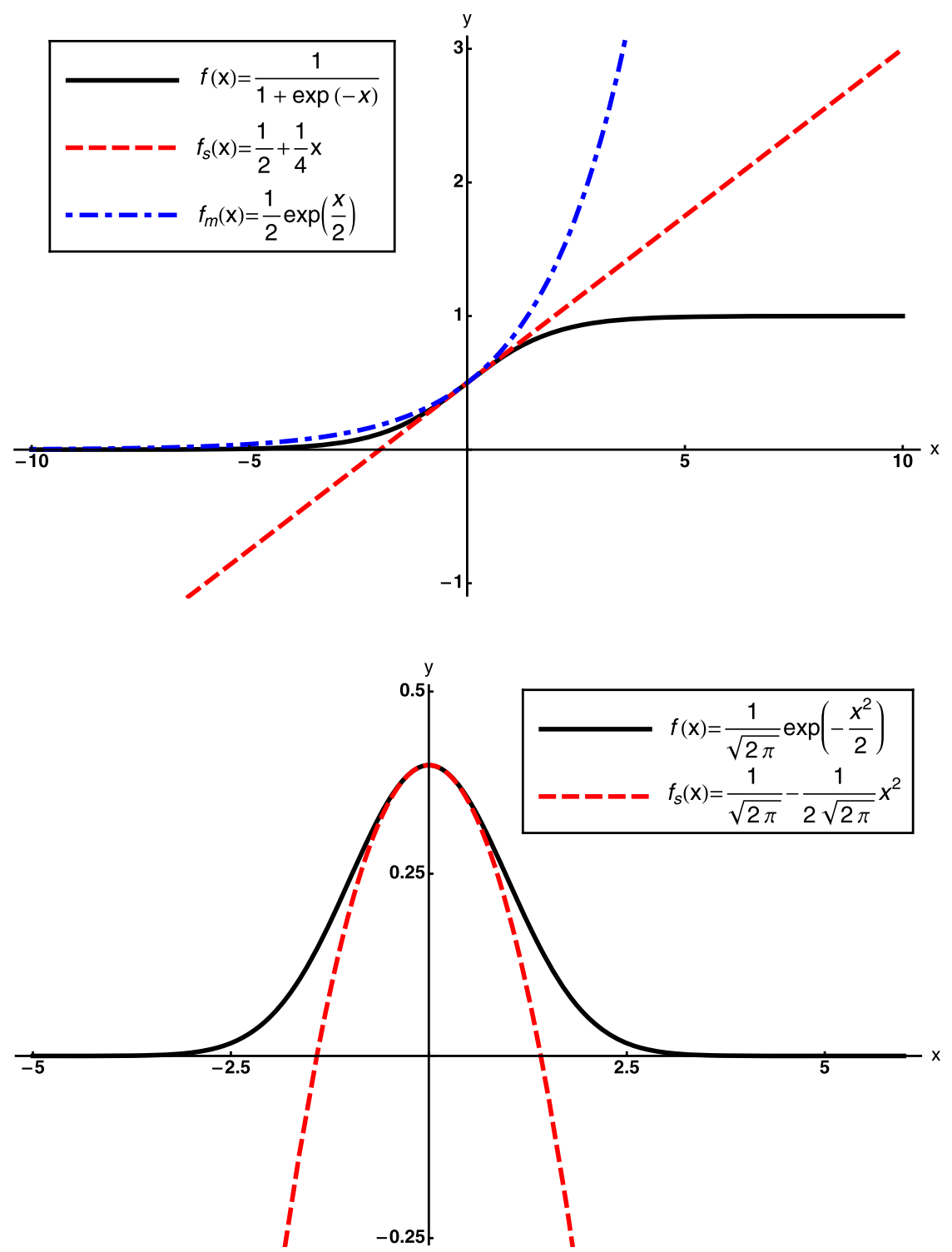

Fig. 4 Gaussian function and its second order standard Taylor expansion. The second order multiplicative Taylor expansion is exact and thus coincides with the original Gaussian function. Recall (26-28)
(ODEs/PDEs). Phenomena driven by some (perhaps implicit) multiplicative mechanism may be more conveniently described in the context of multiplicative calculus than in the standard way. It is beyond the scope of this paper to scrutinize this, but as an illustration we consider the following initial value (ODE) problem:

$$
\left\{\begin{array}{l}
u^{* *}=A, \\
u^{*}(0)=B, \\
u(0)=C,
\end{array}\right.
$$

with $A, B, C>0$ given constants. A straightforward computation, using (4), yields the following unique solution (the multiplicative counterpart of a parabola):

$u(x)=\exp \left(\frac{1}{2} a x^{2}+b x+c\right)$ in which $a=\ln A, b=\ln B, c=\ln C$. Qualitative behaviour is governed by the convexity parameter $A$, with $0<A<1$ producing bounded (Gaussian) solutions, $A=1$ unilaterally unbounded exponential solutions, and $A>1$ bilaterally unbounded solutions. In particular this explains the observation on the coincidence of a Gaussian function and its second order multiplicative Taylor expansion, recall p. 67.

As a second example, let us consider a multiplicative counterpart of the heat equation (PDE):

$\begin{cases}u_{t}^{*}=\Delta^{*} u & \text { for }(x, t) \in \mathbb{R}^{n} \times \mathbb{R}^{+}, \\ u(x, 0)=f(x) & \text { for } x \in \mathbb{R}^{n},\end{cases}$

in which we use multiplicative derivation with respect to both the evolution parameter $t \in \mathbb{R}_{0}^{+}$, i.e. $u_{t}^{*}=\partial_{t}^{*} u$, as well as with respect to the (Cartesian) coordinates $x \in \mathbb{R}^{n}$. The 
multiplicative (*-linear) Laplacian is defined here as

$\Delta^{*}=\exp \circ \Delta \circ \ln$,

cf. (4). Note that, in the commutative case (only), this implies

$\Delta^{*} u=\partial_{x^{1} x^{1}}^{*} u \cdots \partial_{x^{n} x^{n}}^{*} u$.

Again, the solution is straightforward, since in the logarithmic domain the problem reduces to the standard heat equation for $\ln u$ with $\ln f$ as initial condition:

$u(x, t)=\exp \left(\left(\phi_{t} * \ln f\right)(x)\right)$,

in which

$\phi_{t}(x)=\frac{1}{\sqrt{4 \pi t}^{n}} \exp \left(-\frac{\|x\|^{2}}{4 t}\right)$.

Note that if $u$ solves (31), then so does any $*$-linear combination of multiplicative derivatives of $u$.

Equation (31) is a special case of a so-called pseudolinear scale space [16]. Also, the so-called log-Euclidean scale space for diffusion tensor images $[1,9,11,28]$ is governed by a multiplicative system similar to (31), in which case $u$ and $f$ are to be interpreted as positive definite matrix fields, and exp and $\ln$ are the usual extensions applicable to such matrices [11, 24], cf. also Sect. 3.2. Note that in this non-commutative case equivalence of (32) and (33) does not hold, a consequence of the Campbell-Baker-Hausdorff formula:

$$
\begin{aligned}
& \ln (\exp X \exp Y) \\
& \quad=X+Y+\text { commutator terms involving }[X, Y] .
\end{aligned}
$$

\subsection{The Multivariate Case and the Non-commutative Case}

It requires minor efforts to generalize foregoing results to the multivariate case. The PDE example of the previous section is a typical illustration. We will refrain from elaborating on this, but employ such generalizations whenever applicable.

In contrast, as anticipated by (36), extension to the case of non-commutative multiplication is nontrivial, yet highly relevant in modern image analysis practice. For instance, we must account for non-commutative multiplication when handling (positive definite) matrix valued functions, such as diffusion tensor images or strain tensor images. This case has received remarkably little attention. A few results have been provided by Gantmacher [19] and Slavík [33]. It should be noted that Gantmacher's definition, if restricted to scalars, differs from ours. Using the notation $D_{x}$ for multiplicative derivation with respect to $x \in \mathbb{R}$ he defines the multiplicative derivative of a (positive definite, square) matrix field $\boldsymbol{X}: \mathbb{R} \rightarrow \mathbb{M}_{m}^{+}$as follows $\left(\mathbb{M}_{m}\right.$ here denotes the space of real $m \times m$ matrices, and $\mathbb{M}_{m}^{+}$the subspace of positive definite matrices):

$D_{x} \boldsymbol{X}(x)=\boldsymbol{X}^{\prime}(x) \boldsymbol{X}^{-1}(x)$.

Consistency with our notation and definition for the scalar case rather suggests that we use the following definition instead (Slavík [33] discusses various alternatives):

$\boldsymbol{X}^{*}(x)=\exp \left(\boldsymbol{X}^{\prime}(x) \boldsymbol{X}^{-1}(x)\right)$.

One must remain on the alert here, for $X^{\prime} X^{-1}=\ln ^{\prime} X=$ $\boldsymbol{X}^{-1} \boldsymbol{X}^{\prime}$ generically holds only in the commutative case, such as the scalar case $(m=1)$, or the special case whereby $\boldsymbol{X}$ is a linear function in the standard sense of Sect. 2.4, notably (11), recall (36). In other words, for definition (38), and its mirror form, in the context of matrix functions, (3) and the commutative diagram of Fig. 1 do not apply.

Gantmacher also considers the multiplicative integral in a slightly different form. In our case (7) remains applicable, provided we rearrange factors on the right hand side in an unambiguous order, as follows:

$$
\begin{gathered}
* \int_{a}^{b} \boldsymbol{X}(x)^{d x}=\lim _{\triangle x_{i} \rightarrow 0} \boldsymbol{X}\left(\xi_{N}\right)^{\Delta x_{N}} \cdots \boldsymbol{X}\left(\xi_{1}\right)^{\Delta x_{1}} \\
\quad \text { with } \xi_{i} \in\left[x_{i-1}, x_{i}\right] \text { and } x_{0}=a, x_{N}=b .
\end{gathered}
$$

Note that (38) entails a definite choice with respect to the ordering of the factors $\boldsymbol{X}^{\prime}$ and $\boldsymbol{X}^{-1}$ in the multiplicative derivative, which affects the corresponding definition of the antiderivative, (39), as well. Thus we have at least three distinct ways to introduce multiplicative differential and integral calculus in the context of matrix functions, viz. (i) (38) in combination with (39), (ii) the analogous scheme with reverse ordering of $\boldsymbol{X}^{\prime}$ and $\boldsymbol{X}^{-1}$, respectively of the $*$ infinitesimal factors as they occur in the defining limiting procedure of the multiplicative integral, and (iii) the matrix equivalent of the ln/exp-formalism of (3). The first option (i), i.e. (38-39), meets our needs in the example of Sect. 3.1. In Sect. 3.2 we will illustrate the third, in some sense "unbiased" option (iii), which appears to be the natural one in the context of the so-called log-Euclidean paradigm for diffusion tensor imaging $[1,9,11,28]$.

\section{Examples}

\subsection{Lagrangian Strain Analysis of the Myocardium}

Cardiac strain analysis can be based on any imaging protocol and image analysis algorithm that produces an accurate estimate of the gradient velocity tensor field of material points in the myocardium as a function of position and time 
in the image sequence. An analytical procedure for this has been proposed elsewhere [2, 12, 17], based on tagging magnetic resonance imaging, a technique originally proposed by Zerhouni et al. [36], and incrementally improved to its current state of the art, including volumetric tagging [30, 32].

In the following example we sketch the analytical procedure underlying cardiac strain analysis, recasting it in a multiplicative framework from the outset. At the same time this shows how to extend the scalar framework to the case of (positive definite) matrix valued functions. Detailed definitions and proofs (based on standard calculus) can be found elsewhere [12].

The velocity gradient tensor, $\boldsymbol{L}$, with components ${ }^{1} L_{\beta}^{\alpha}$ relative to a coordinate frame, relates the rate of change of a momentary infinitesimal material line element $d \dot{x}^{\alpha}$ to the line element $d x^{\beta}$ itself. From $d \dot{x}^{\alpha}=d v^{\alpha}$ it follows, using the chain rule, that ${ }^{2}$

$d \dot{x}^{\alpha}=L_{\beta}^{\alpha} d x^{\beta}$

with $L_{\beta}^{\alpha}=\frac{\partial v^{\alpha}}{\partial x^{\beta}} \quad(\alpha, \beta=1, \ldots, n)$.

If $X=x\left(X, t_{0}\right)$ denotes the position of a material point at a fiducial moment $t_{0}$, and $x=x(X, t)$ the position of the same material point at some later moment in the cardiac cycle, $t \geq t_{0}$, then relative tissue deformation can be described by a smooth mapping $x\left(X, t ; t_{0}\right)$. We considering this as a function of $X$ and $t$. The associated differential map, called the deformation tensor field, is characterized by the Jacobian matrix $\boldsymbol{F}$, with components

$F_{i}^{\alpha}=\frac{\partial x^{\alpha}}{\partial X^{i}}$.

By virtue of the chain rule, the relation between deformation and velocity gradient tensors, (40) and (41), is given by the first order ODE [19]

$\dot{\boldsymbol{F}}=\boldsymbol{L} \boldsymbol{F}$,

subject to an initial condition. ${ }^{3}$ The multiplicative nature of the evolution of $\boldsymbol{F}$ is apparent from (42), reflecting the fact that concatenations of (infinitesimal) deformations correspond to multiplications (respectively multiplicative integration) of the associated Jacobians.

The simplicity of (42) is, however, deceptive. The essential complication arises due to the fact that $\boldsymbol{L}$ is a nonstationary matrix (as a result of which $[\boldsymbol{L}(s), \boldsymbol{L}(t)] \neq 0$ for

\footnotetext{
${ }^{1}$ Upper indices serve as row indices, lower indices as column indices.

${ }^{2}$ The Einstein summation convention applied here will be used henceforth.

${ }^{3}$ We suppress the spatial dependence of the Jacobian, concentrating on its $\left(t, t_{0}\right)$-dependence, taking $t$ as our variable and $t_{0}$ as a fixed parameter.
}

$s \neq t$, causing complications due to (36)). It can be shown, using standard calculus ${ }^{4}[12,19]$, that the solution to (42) with initial condition $\boldsymbol{F}\left(t=t_{0}, t_{0}\right)=\boldsymbol{I}$ is given by

$\boldsymbol{F}\left(t, t_{0}\right)=* \int_{t_{0}}^{t} \exp (\boldsymbol{L}(\tau) d \tau)$,

recall (39). This nontrivial explicit solution clearly confirms the multiplicative nature of the problem already foreseen in its implicit differential form, (42). One should therefore expect that the problem would have been much simpler if it had been stated in multiplicative differential form from the outset. Indeed, if we define the corresponding multiplicative derivative according to (38), then (42) simplifies to

$\boldsymbol{F}^{*}=\exp (\boldsymbol{L}) \quad$ with $\boldsymbol{F}\left(t=t_{0}, t_{0}\right)=\boldsymbol{I}$,

immediately yielding the solution via antiderivation, ${ }^{5}$ (43).

Several properties of the deformation tensor are manifest in multiplicative representation. For instance, for square matrices $\boldsymbol{A}, \boldsymbol{B}$, one has

i. $\operatorname{det} \boldsymbol{A} \boldsymbol{B}=\operatorname{det} \boldsymbol{A} \operatorname{det} \boldsymbol{B}$,

ii. $\operatorname{det}(\boldsymbol{I}+\epsilon \boldsymbol{A})=1+\epsilon \operatorname{tr} \boldsymbol{A}+\mathcal{O}\left(\epsilon^{2}\right)$, and

iii. $\operatorname{det} \exp \boldsymbol{A}=\exp \operatorname{tr} \boldsymbol{A}$.

Consequently,

$\operatorname{det} \boldsymbol{F}\left(t, t_{0}\right)=* \int_{t_{0}}^{t} \exp (\operatorname{tr} \boldsymbol{L}(\tau) d \tau)$,

consistent with the multiplicative integral introduced for the scalar case, (7). This confirms, in particular, that a divergence free velocity field $(\operatorname{tr} \boldsymbol{L}=\operatorname{div} \boldsymbol{v}=0)$ preserves volumes: $\operatorname{det} \boldsymbol{F}\left(t, t_{0}\right)=1$. Furthermore, from (36) it follows that $\exp \boldsymbol{A} \exp \boldsymbol{B}=\exp (\boldsymbol{A}+\boldsymbol{B})$ if $[\boldsymbol{A}, \boldsymbol{B}]=0$, whence for a stationary velocity field $\left(\boldsymbol{L}(t)=\boldsymbol{L}_{0}\right.$ time independent) (43) directly yields $\boldsymbol{F}\left(t, t_{0}\right)=\exp \left(\left(t-t_{0}\right) \boldsymbol{L}_{0}\right)$. However, motion inducing myocardial deformation is typically highly nonstationary, so that this stationary approximation will not provide a good approximation for (43).

The multiplicative integral suggests a straightforward numerical approximation akin to its standard counterpart, simply by using (39) and (43) without limiting procedure (with constant time steps $\Delta \tau_{i}=\Delta \tau$ induced by the frame rate of the image sequence, say). Results reported elsewhere [12], as well as Figs. 5 and 6, have been obtained in this way.

The deformation tensor field immediately yields the Lagrangian strain tensor field [26] (also known as the Green strain tensor field [23]):

$\boldsymbol{E}=\frac{1}{2}\left(\boldsymbol{F}^{\mathrm{T}} \boldsymbol{F}-\boldsymbol{I}\right)$.

\footnotetext{
${ }^{4}$ The proof is not difficult, but far from trivial.

${ }^{5}$ Note that the multiplicative rate of change $\boldsymbol{F}^{*}$ is somewhat peculiar from a dimensional analysis point of view, unlike the corresponding absolute change $* d \boldsymbol{F}=\boldsymbol{F}^{* d t}$.
} 


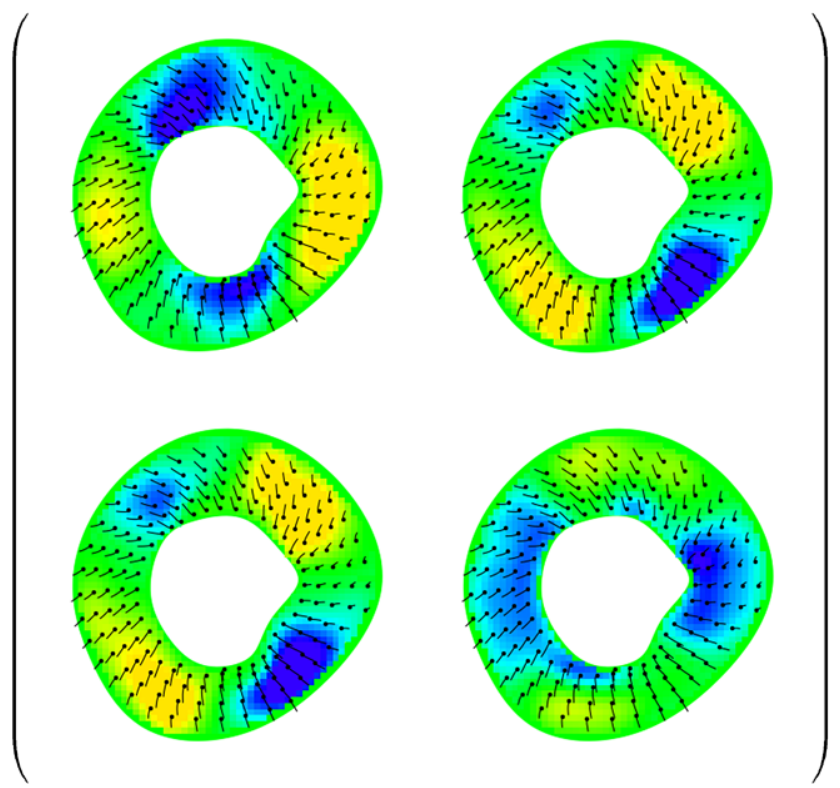

Fig. 5 Strain tensor field evaluated for a healthy volunteer at end-systole $t=t_{1}$ relative to end-diastole $t=t_{0}$. The matrix shows the four (three independent) Cartesian components $E_{i j}\left(x, y, t_{1}\right), i, j=1,2$, with row index $i$ and column index $j$, at each point $(x, y) \in \Omega \subset \mathbb{Z}^{2}$ of a short-axis cross-section. Fiducial tissue markers have been overlayed together with their trajectories starting at $t=t_{0}$ to visualize the evolution of deformation. The pixel value at a given location in the actual tensor valued image is the $2 \times 2$ matrix obtained by collecting the entries from corresponding points in the component images displayed in the matrix above. Recall (43) and (46)

The field $\boldsymbol{E}$ vanishes identically under isometric deformations, thus capturing genuinely nonrigid deformations.

Figures 5 and 6 illustrate the Lagrangian strain tensor field for a 2-dimensional short-axis cross-section of the left ventricle at end-systole $\left(t=t_{1}\right)$ relative to end-diastole $\left(t=t_{0}\right)$. For more details, cf. Van Assen et al. [3].

\subsection{Multiscale Representation of Positive Definite Matrix Fields}

The so-called log-Euclidean paradigm provides an example of a representation that takes positivity into account $a$ priori. It has been proposed in the context of symmetric positive definite diffusion tensor images $[1,9,28]$, although it is in itself of a more generic nature. Here we consider the paradigm in the context of multiscale representations of diffusion tensor images, as introduced elsewhere [11].

We denote a diffusion tensor image by $\boldsymbol{X}: \mathbb{R}^{n} \rightarrow \mathbb{S}_{n}^{+}$, where $\mathbb{S}_{n}^{+} \subset \mathbb{S}_{n} \subset \mathbb{M}_{n}$ denotes the set of $\mathbb{R}$-valued symmetric positive definite $n \times n$ matrices, $\mathbb{S}_{n}$ the set of $\mathbb{R}$-valued symmetric $n \times n$ matrices, and $\mathbb{M}_{n}$ the set of all $\mathbb{R}$-valued $n \times n$ matrices. Its pointwise inverse is $X^{\text {inv }}: \mathbb{R}^{n} \rightarrow \mathbb{S}_{n}^{+}$, so that $\left(\boldsymbol{X}^{\text {inv }} \boldsymbol{X}\right)(x)=\left(\boldsymbol{X} \boldsymbol{X}^{\text {inv }}\right)(x)=\boldsymbol{I}$, the identity matrix, at each point $x \in \mathbb{R}^{n}$. $C^{\omega}\left(\mathbb{R}^{n}, \mathbb{M}_{n}\right)$ denotes the class of analytical functions $\boldsymbol{X}: \mathbb{R}^{n} \rightarrow \mathbb{M}_{n}$. Self-explanatory definitions hold for $C^{\omega}\left(\mathbb{R}^{n}, \mathbb{S}_{n}^{+}\right) \subset C^{\omega}\left(\mathbb{R}^{n}, \mathbb{S}_{n}\right) \subset C^{\omega}\left(\mathbb{R}^{n}, \mathbb{M}_{n}\right)$.

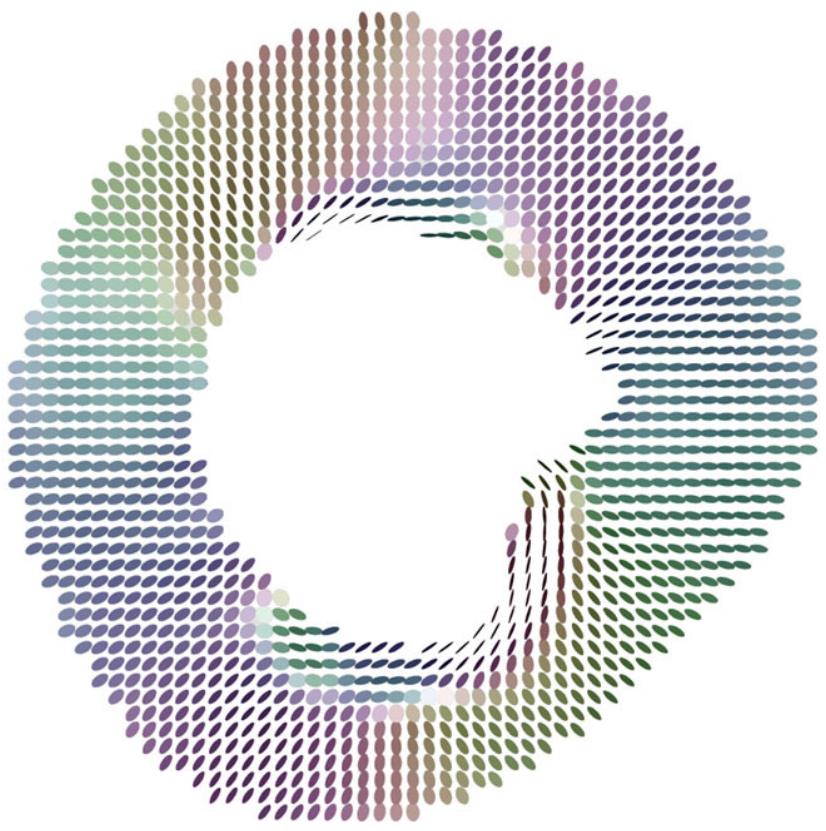

Fig. 6 The same strain tensor field as in Fig. 5, but with each tensor displayed as an ellipsoidal gauge figure reflecting the eigensystem of the non-negative definite matrix $\boldsymbol{F}^{\mathrm{T}} \boldsymbol{F}=2 \boldsymbol{E}+\boldsymbol{I}$. More precisely, the boundary of each gauge figure is given by the quadric $\boldsymbol{F} \vec{\xi} \cdot \boldsymbol{F} \vec{\xi}=$ constant, with $\vec{\xi}=(\xi, \eta)$, and $\boldsymbol{F}$ evaluated at the corresponding spatiotemporal base point $(x, y, t)$. Hue emphasizes main direction, while purity is a measure of anisotropy (with white corresponding to an isotropic, i.e. circular figure)

The scale space representation of $\boldsymbol{X} \in C^{\omega}\left(\mathbb{R}^{n}, \mathbb{S}_{n}^{+}\right)$is generated by the blurring operator (detailed below)

$\mathcal{F}: C^{\omega}\left(\mathbb{R}^{n}, \mathbb{S}_{n}^{+}\right) \times \mathbb{R}^{+} \rightarrow C^{\omega}\left(\mathbb{R}^{n}, \mathbb{S}_{n}^{+}\right):(\boldsymbol{X}, t) \mapsto \mathcal{F}(\boldsymbol{X}, t)$,

with $\mathcal{F}(\boldsymbol{X}, 0)=\boldsymbol{X}$ for all $\boldsymbol{X} \in C^{\omega}\left(\mathbb{R}^{n}, \mathbb{S}_{n}^{+}\right)$. We use the shorthand notation $\boldsymbol{X}_{t} \equiv \mathcal{F}(\boldsymbol{X}, t)$. The isotropic Gaussian scale space kernel in $n$ dimensions is given by (35).

Elsewhere it has been argued that the closure requirement

$\mathcal{F}(\boldsymbol{X}, t)^{\mathrm{inv}}=\mathcal{F}\left(\boldsymbol{X}^{\text {inv }}, t\right)$,

in other words, the condition that blurring and inversion should commute, naturally leads to the log-Euclidean paradigm [11].

Recall that the exponential map exp : $\mathbb{M}_{n} \rightarrow \mathrm{GL}_{n}$ maps a general $n \times n$ matrix to a nonsingular matrix, i.e. an element of the general linear group $[18,19,27]$. For later convenience we define $\mathbb{M}_{n}^{+}=\exp \left(\mathbb{M}_{n}\right) \subset \mathrm{GL}_{n}$. For our purpose it suffices to consider elements of $\mathbb{S}_{n} \subset \mathbb{M}_{n}$, which are diagonalizable with real eigenvalues, in which case the range of the exponential map equals $\exp \left(\mathbb{S}_{n}\right)=\mathbb{S}_{n}^{+}$. So we will employ the prototype

$\exp : \mathbb{S}_{n} \rightarrow \mathbb{S}_{n}^{+}: \boldsymbol{A} \mapsto \exp \boldsymbol{A}$. 


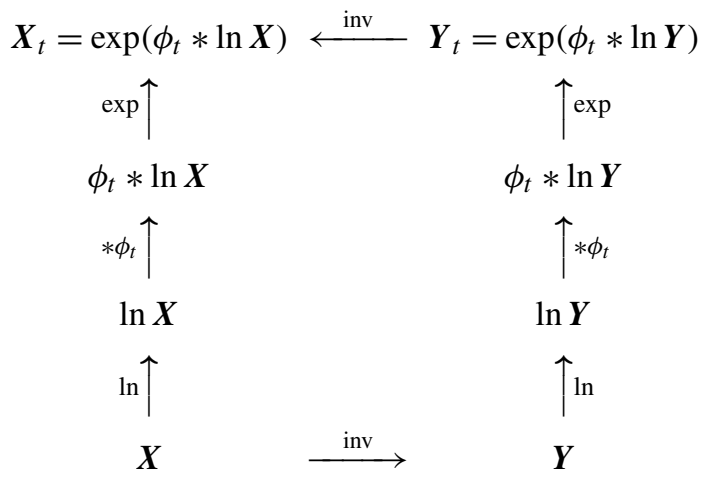

Fig. 7 Commuting diagram for blurring and inversion

An operational representation of a general analytical matrix function is given by Sylvester's formula ${ }^{6}[5-7,24]$ :

$F(\boldsymbol{A}) \stackrel{\text { def }}{=} \sum_{i=1}^{m} F\left(\lambda_{i}\right) \boldsymbol{A}_{i}$,

in which the $\lambda_{i}, i=1, \ldots, m \leq n$, are all distinct eigenvalues of $\boldsymbol{A}$. In (50) the left hand side-with intentional abuse of notation, or "argument overloading"-is defined by virtue of the analytical scalar function $F \in C^{\omega}(\mathbb{R}, \mathbb{R})$ on the right hand side, i.c. $F \equiv \exp$, and the so-called Frobenius covariants are given by

$\boldsymbol{A}_{i}=\prod_{j=1, j \neq i}^{m} \frac{1}{\lambda_{i}-\lambda_{j}}\left(\boldsymbol{A}-\lambda_{j} \boldsymbol{I}\right)$.

It is conventionally understood that an empty product (which occurs in the most degenerate case in which all eigenvalues coincide, i.e. $m=1$ ) evaluates to the unit matrix.

The logarithmic map, restricted to $\mathbb{S}_{n}^{+}$, has prototype

$\ln : \mathbb{S}_{n}^{+} \rightarrow \mathbb{S}_{n}: \boldsymbol{B} \mapsto \ln \boldsymbol{B}$.

It is the unique inverse of the exponential map on $\mathbb{S}_{n}$ : $\ln \left(\mathbb{S}_{n}^{+}\right)=\mathbb{S}_{n}$. Equations (50-51) are applicable with $F \equiv \ln$.

Figure 7 shows the multiscale representation consistent with the closure property, (48). Indeed, if $\boldsymbol{X} \in C^{\omega}\left(\mathbb{R}^{n}, \mathbb{S}_{n}^{+}\right)$, then $\boldsymbol{X}_{t}=\mathcal{F}(\boldsymbol{X}, t)$ constructed according to

$\mathcal{F}(\boldsymbol{X}, t)=\exp \left(\phi_{t} * \ln \boldsymbol{X}\right)$,

satisfies the desired commutativity property, (48). This follows immediately by inspection of Fig. 7 and (53), using the identities

$\exp (-\boldsymbol{A})=(\exp \boldsymbol{A})^{\mathrm{inv}} \quad$ and $\quad \ln \boldsymbol{B}^{\text {inv }}=-\ln \boldsymbol{B}$,

${ }^{6}$ Generically one expects $m=n$ a.e. within the image domain. for $\boldsymbol{A} \in \mathbb{S}_{n}, \boldsymbol{B} \in \mathbb{S}_{n}^{+}$. For illustrations of DTI blurring, cf. Florack and Astola [11].

Formulae for standard differential calculus applied to (53) are highly nontrivial, cf. the explicit computations of first and second order standard derivatives by Florack and Astola [11]. The log-Euclidean paradigm suggests the following way to introduce multiplicative derivation for the non-commutative case, recall the three options discussed in Sect. 2.8:

$\boldsymbol{X}^{*} \stackrel{\text { def }}{=} \exp \left((\ln \boldsymbol{X})^{\prime}\right)$

for the one-dimensional case. This is similar to (3) for the scalar case, but recall that in (55) exp and $\ln$ are the matrix exponential and logarithm, respectively. For the multivariate case this leads to the following operationalization of the multiplicative gradient of $\boldsymbol{X}_{t}=\mathcal{F}(\boldsymbol{X}, t)$, recall (53):

$\partial_{i}^{*} \boldsymbol{X}_{t} \stackrel{\text { def }}{=} \exp \left(\partial_{i} \phi_{t} * \ln \boldsymbol{X}\right)$.

Equation (56) is consistent with the Gaussian scale space paradigm given by (31) and (32), in which standard diffusion and Gaussian convolution are now applied componentwise to matrix entries via the $\ln / \exp$ detour. The (hypothetical) infinite-resolution limit, $t \rightarrow 0$, establishes the correspondence between (56) and the non-operational (ill-posed) "infinitesimal" one [10], i.e. the multivariate counterpart of (55):

$\partial_{i}^{*} \boldsymbol{X} \stackrel{\text { def }}{=} \exp \left(\partial_{i} \ln \boldsymbol{X}\right)$.

This definition of multiplicative derivation thus seems to fit naturally with the log-Euclidean paradigm $[1,9,11,28]$. Adhering to this definition, log-Euclidean blurring can be seen as the multiplicative counterpart of a standard diffusion process, i.e. the counterpart of (31-35) for positive symmetric matrix-valued functions. See Fig. 8 for an example of multiplicative diffusion for regularizing tractography.

As a final remark it should be noted that the logEuclidean paradigm has been discussed here as an instance of a multiplicative calculus for positive definite matrix fields, based on the standard matrix product. In this context, it has been argued that (55-57) are choices, on a par with alternatives such as (38) and its mirror form. If one restricts oneself to the log-Euclidean paradigm as the axiom of choice, it may be more convenient to consider the specific, symmetric product operator $\bullet$, given by $\boldsymbol{A} \bullet \boldsymbol{B}=\exp (\ln \boldsymbol{A}+\ln \boldsymbol{B})$, and consider the corresponding multiplicative calculus from the outset. (Due to commutativity this may greatly simplify the analysis.) This, and other symmetric matrix products, together with their implications for differential calculus, have been proposed in the literature, and may likewise provide points of departure for useful instances of multiplicative calculus, depending on context, cf. Burgeth et al. [8]. 

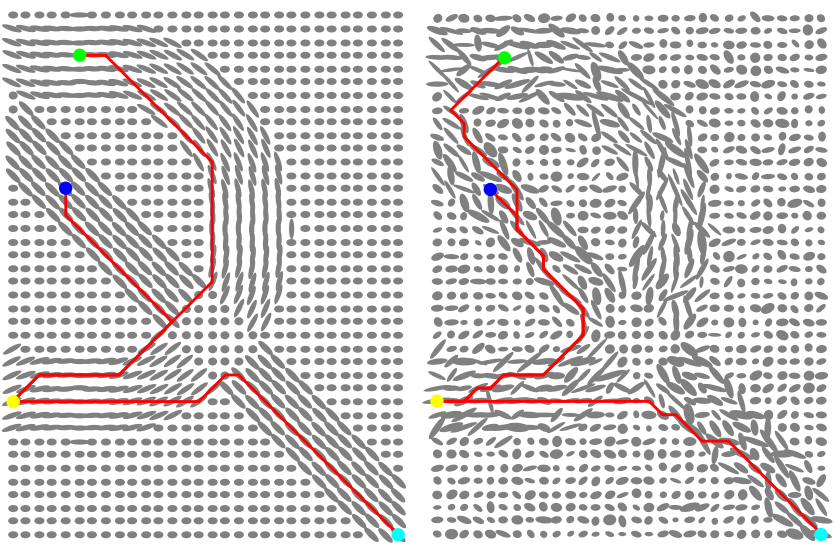

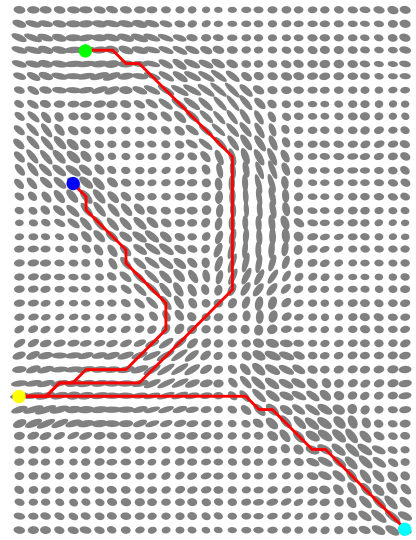

Fig. 8 Two-dimensional synthetic images illustrating a positive symmetric tensor field in terms of ellipsoidal glyphs (principal axes and radii reflect eigendirections and corresponding eigenvalues). Overlayed are some fixed end-point geodesics obtained by applying Dijkstra's shortest path algorithm, in which the tensor field itself is interpreted as the dual Riemannian metric for defining distances. This complies with the Riemannian rationale for geodesic tractography in

\section{Conclusion and Discussion}

Multiplicative calculus and its applications to biomedical image analysis raises many important questions not addressed in this short paper. For instance, since image intrinsic properties must be coordinate independent, a question arises about its implications for the construction of image differential invariants [13-15] and tensor calculus. A second question pertains to the extension of standard variational techniques for image optimization problems to the multiplicative case. How to set up such a framework rigorously? In biomedical image analysis such a framework would have the intrinsic advantage that positivity of solutions would be guaranteed a priori. Additional questions arise in the context of (non-commutative) matrix fields. Which of the three proposed options for multiplicative differential calculus (if any) is the most natural one in a given application context, what are their mutual relations, how do they relate to standard differential calculus, and, in the log-Euclidean case of (57), what does the corresponding antiderivative look like?

Despite major open questions it has been argued that multiplicative calculus provides a natural framework for biomedical image analysis, particularly in problems in which positive images or positive definite matrix fields and positivity preserving operators are of interest. We therefore believe that this subject is of broad interest. However, it seems that many fundamental problems have not been addressed in the mathematical literature sofar, especially regarding the non-commutative case. This is an impediment for progress in biomedical image analysis.

Examples have been given in the context of cardiac strain analysis and diffusion tensor imaging to illustrate the rele- diffusion tensor imaging [25, 29]. The left image shows the result for the originally synthesized, smooth image. The middle image shows the result of the same algorithm after the image has been perturbed by pixel-uncorrelated noise. The right image demonstrates the regularizing effect of log-Euclidean blurring, (53), and its effect on the performance of the algorithm

vance of multiplicative calculus in biomedical image analysis, and to support our recommendation for further investigation into practical as well as fundamental issues.

Acknowledgements The Netherlands Organisation for Scientific Research (NWO) is gratefully acknowledged for financial support. We thank Shufang Liu for conducting a literature study for us. Jos Westenberg provided tagging MRI data from which the strain tensor fields illustrated in Figs. 5-6 have been generated. Laura Astola generated the synthetical data and tractography results shown in Fig. 8.

Open Access This article is distributed under the terms of the Creative Commons Attribution Noncommercial License which permits any noncommercial use, distribution, and reproduction in any medium, provided the original author(s) and source are credited.

\section{References}

1. Arsigny, V., Fillard, P., Pennec, X., Ayache, N.: Log-Euclidean metrics for fast and simple calculus on diffusion tensors. Magn. Reson. Med. 56(2), 411-421 (2006)

2. Assen, H.V., Florack, L., Suinesiaputra, A., Westenberg, J., ter Haar Romeny, B.: Purely evidence based multiscale cardiac tracking using optic flow. In: Miller, K., Paulsen, K.D., Young, A.A., Nielsen, P.M.F. (eds.) Proceedings of the MICCAI Workshop on Computational Biomechanics for Medicine II, Brisbane, Australia, October 29, 2007, pp. 84-93 (2007)

3. Assen, H.C.V., Florack, L.M.J., Simonis, F.F.J., Westenberg, J.J.M., Strijkers, G.J.: Cardiac strain and rotation analysis using multi-scale optical flow. In: Wittek, A., Nielsen, P.M.F. (eds.) Proceedings of the MICCAI Workshop on Computational Biomechanics for Medicine V, Beijing, China, September 24, 2010, pp. 89-100 (2010)

4. Bashirov, A.E., Kurpinar, E.M., Özyapici, A.: Multiplicative calculus and its applications. J. Math. Anal. Appl. 337, 36-48 (2008)

5. Bellman, R.: Introduction to Matrix Analysis, 2nd edn. Classics in Applied Mathematics, vol. 19. SIAM, Philadelphia (1997) 
6. Buchheim, A.: On the theory of matrices. Proc. Lond. Math. Soc. 16, 63-82 (1884)

7. Buchheim, A.: An extension of a theorem of professor Sylvester's relating to matrices. Philos. Mag. 22(135), 173-174 (1886)

8. Burgeth, B., Didas, S., Florack, L., Weickert, J.: A generic approach to diffusion filtering of matrix-fields. Computing 81(2-3), 179-197 (2007)

9. Fillard, P., Pennec, X., Arsigny, V., Ayache, N.: Clinical DT-MRI estimation, smoothing, and fiber tracking with log-Euclidean metrics. IEEE Trans. Med. Imag. 26(11) (2007)

10. Florack, L.M.J.: Image Structure, Computational Imaging and Vision Series, vol. 10. Kluwer Academic, Dordrecht (1997)

11. Florack, L.M.J., Astola, L.J.: A multi-resolution framework for diffusion tensor images. In: Fernández, S.A., de Luis Garcia, R. (eds.) CVPR Workshop on Tensors in Image Processing and Computer Vision, Anchorage, Alaska, USA, June 24-26, 2008. IEEE Press, New York (2008). Digital proceedings

12. Florack, L., van Assen, H.: A new methodology for multiscale myocardial deformation and strain analysis based on tagging MRI. Int. J. Biomed. Imaging (2010). doi:10.1155/ 2010/341242. URL http://www.hindawi.com/journals/ijbi/2010/ 341242.html

13. Florack, L.M.J., Haar Romeny, B.M.T., Koenderink, J.J., Viergever, M.A.: Scale and the differential structure of images. Image Vis. Comput. 10(6), 376-388 (1992)

14. Florack, L.M.J., Haar Romeny, B.M.T., Koenderink, J.J., Viergever, M.A.: Cartesian differential invariants in scale-space. J. Math. Imaging Vis. 3(4), 327-348 (1993)

15. Florack, L.M.J., Haar Romeny, B.M.T., Koenderink, J.J., Viergever, M.A.: General intensity transformations and differential invariants. J. Math. Imaging Vis. 4(2), 171-187 (1994)

16. Florack, L.M.J., Maas, R., Niessen, W.J.: Pseudo-linear scalespace theory. Int. J. Comput. Vis. 31(2-3), 247-259 (1999)

17. Florack, L., van Assen, H., Suinesiaputra, A.: Dense multiscale motion extraction from cardiac cine MR tagging using HARP technology. In: Niessen, W., Westin, C.F., Nielsen, M. (eds.) Proceedings of the 8th IEEE Computer Society Workshop on Mathematical Methods in Biomedical Image Analysis, Held in Conjunction with the IEEE International Conference on Computer Vision, Rio de Janeiro, Brazil, October 14-20, 2007 (2007). Digital proceedings by Omnipress

18. Fung, T.C.: Computation of the matrix exponential and its derivatives by scaling and squaring. Int. J. Numer. Methods Eng. 59, 1273-1286 (2004)

19. Gantmacher, F.R.: The Theory of Matrices. American Mathematical Society, Providence (2001)

20. Gill, R.D., Johansen, S.: A survey of product-integration with a view toward application in survival analysis. Ann. Stat. 18, 15011555 (1990)

21. Grossman, M., Katz, R.: Non-Newtonian Calculus. Lee Press, Pigeon Cove (1972)

22. Guenther, R.A.: Product integrals and sum integrals. Int. J. Math. Educ. Sci. Technol. 14(2), 243-249 (1983)

23. Haupt, P.: Continuum Mechanics and Theory of Materials. Springer, Berlin (2002)

24. Higham, N.J.: Functions of Matrices: Theory and Computation. SIAM, Philadelphia (2008)

25. Lenglet, C., Deriche, R., Faugeras, O.: Inferring white matter geometry from diffusion tensor MRI: Application to connectivity mapping. In: Pajdla, T., Matas, J. (eds.) Proceedings of the Eighth European Conference on Computer Vision, Prague, Czech Republic, May 2004. Lecture Notes in Computer Science, vol. 30213024, pp. 127-140. Springer, Berlin (2004)

26. Marsden, J.E., Hughes, T.J.R.: Mathematical Foundations of Elasticity. Dover, Mineola (1994)
27. Moler, C., Van Loan, C.: Nineteen dubious ways to compute the exponential of a matrix. SIAM Rev. 20(4), 801-836 (1978)

28. Pennec, X., Fillard, P., Ayache, N.: A Riemannian framework for tensor computing. Int. J. Comput. Vis. 66(1), 41-66 (2006)

29. Prados, E., Soatto, S., Lenglet, C., Pons, J.P., Wotawa, N., Deriche, R., Faugeras, O.: Control theory and fast marching techniques for brain connectivity mapping. In: Proceedings of the IEEE Computer Society Conference on Computer Vision and Pattern Recognition, New York, USA, June 2006, vol. 1, pp. 1076-1083. IEEE Computer Society, Los Alamitos (2006)

30. Rutz, A.K., Ryf, S., Plein, S., Boesiger, P., Kozerke, S.: Accelerated whole-heart 3D CSPAMM for myocardial motion quantification. Magn. Reson. Med. 59(4), 755-763 (2008)

31. Rybaczuk, M., Kędzia, A., Zieliński, W.: The concept of physical and fractal dimension II. the differential calculus in dimensional spaces. Chaos Solitons Fractals 12, 2537-2552 (2001)

32. Ryf, S., Spiegel, M.A., Gerber, M.P.B.: Myocardial tagging with 3D-CSPAMM. J. Magn. Reson. Imaging 16(3), 320-325 (2002)

33. Slavík, A.: Product Integration, Its History and Applications. Matfyzpress, Prague (2007)

34. Stanley, D.: A multiplicative calculus. PRIMUS, Probl. Resour. Issues Math. Undergrad. Stud. IX(4), 310-326 (1999)

35. Volterra, V.: Sulle equazioni differenziali lineari. Rend. Acad. Lincei, Ser. 4 3, 393-396 (1887)

36. Zerhouni, E.A., Parish, D.M., Rogers, W.J., Yang, A., Shapiro, E.P.: Human heart: tagging with MR imaging-a method for noninvasive assessment of myocardial motion. Radiology 169(1), 5963 (1988)

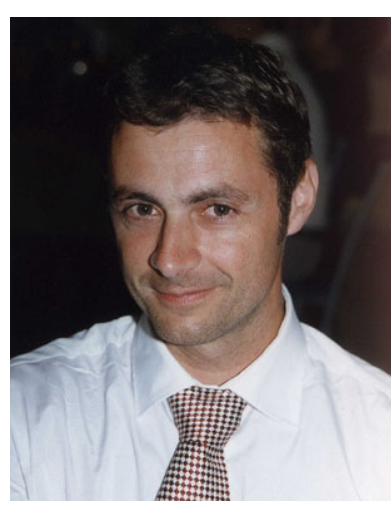

Luc Florack received his M.Sc. degree in theoretical physics in 1989, and his Ph.D. degree cum laude in 1993 with a thesis on image structure under the supervision of professor Max Viergever and professor Jan Koenderink, both from Utrecht University, The Netherlands. During the period 19941995 he was an ERCIM/HCM research fellow at INRIA SophiaAntipolis, France, with professor Olivier Faugeras, and at INESC Aveiro, Portugal, with professor Antonio Sousa Pereira. In 1996 he was an assistant research professor at DIKU, Copenhagen, Denmark, with professor Peter Johansen, on a grant from the Danish Research Council. In 1997 he returned to Utrecht University, were he became an assistant research professor at the Department of Mathematics and Computer Science. In 2001 he moved to Eindhoven University of Technology, Department of Biomedical Engineering, where he became an associate professor in 2002. In 2007 he was appointed full professor at the Department of Mathematics and Computer Science, and established the chair of Mathematical Image Analysis, but retained a parttime professorship at the former department. His research covers mathematical models of structural aspects of signals, images, and movies, particularly multiscale and differential geometric representations and their applications, with a focus on complex magnetic resonance images for cardiological and neurological applications. In 2010, with support of the Executive Board of Eindhoven University of Technology, he established the Imaging Science \& Technology research group (IST/e), a cross-divisional collaboration involving several academic groups on image acquisition, biomedical and mathematical image analysis, visualization and visual analytics. 


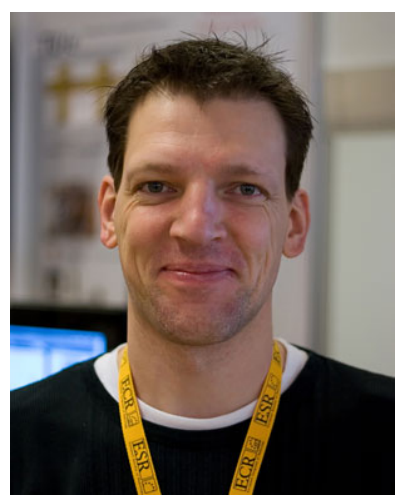

Hans van Assen received his M.Sc. degree in applied physics in 1995 from Delft University of Technology, and his Ph.D. degree in 2006 with a thesis on cardiac segmentation based on statistical modeling under the supervision of professor Hans Reiber, from Leiden University, The Netherlands. During the period 1996-2000 he was a research scientist at LKEB in the Leiden University Medical Center, The Netherlands. In 2005 he moved to Eindhoven University of Technology, Department of Biomedical
Engineering. Until 2008 he was a post-doctoral fellow and since 2008 he has held a position as an assistant professor Cardiac Image Analysis in the Biomedical Image Analysis group headed by professor Bart ter Haar Romeny. His research involves cardiac motion and deformation analysis in relation to the automatic diagnosis of cardiac pathology, and segmentation and image guidance of interventions pertaining to, e.g., atrial fibrillation. In 2010, he was a co-applicant of the grant that started the Imaging Science \& Technology research group (IST/e), a cross-divisional collaboration involving several academic groups on image acquisition, biomedical and mathematical image analysis, visualization and visual analytics. Recently, he received two grants from STW (Dutch Technology Foundation) related to both his research lines. 